\title{
Modeling dynamics of fast food and obesity for evaluating the peer pressure effect and workout impact
}

\author{
Salma M. Al-Tuwairqi ${ }^{*}$ (1) and Reem T. Matbouli
}

\section{"Correspondence:}

saltuwairqi@kau.edu.sa

'Mathematics Department, King Abdulaziz University, Jeddah, Saudi Arabia

\section{Springer}

\begin{abstract}
In recent years, chronic diseases, such as high blood pressure, diabetes, heart attack, and cancer, have increased around the world. Obesity is a common factor that makes individuals susceptible to these diseases. One reason for excessive weight gain is the frequent consumption of fast food. This study examined the impact that fast food has on obesity by analyzing the influence of peer pressure on fast food consumption and the role that exercise plays in weight gain. Two mathematical models were proposed to study each factor separately. The models demonstrated the dynamics of individuals transitioning from one weight class to another according to their body mass index (BMI). The models were governed by systems of nonlinear ordinary differential equations. A qualitative approach for analyzing the systems was utilized, and the numerical simulations supported the qualitative results. The results of this study may contribute to the development of strategies for controlling obesity. In particular, the models showed the importance of resisting peer pressure that drives individuals to eat fast food meals, as well as maximizing the role that quitters can play in convincing obese individuals to stop consuming fast food meals. In addition to the two previous strategies, the research revealed that physical activity has a significant role in reducing weight.
\end{abstract}

Keywords: Mathematical model; Obesity; Dynamical system; Global stability; Local stability

\section{Introduction}

Obesity is a condition defined by increased levels of fat in the human body. Individuals are classified according to their weight using the global body mass index (BMI). This measurement is calculated by dividing the body mass (weight) over the square of the body height. If the output is less than 18.5 , then the person is classified as underweight. The normal and ideal weight for a person occurs if the BMI is between 18.5 and 24.9. If the BMI is between 25 and 30, then the person is overweight and is more likely to become obese. Once the BMI score is more than 30, then the person is considered obese.

Many factors may contribute to weight gain, such as environment, culture, metabolism, and genes. However, the primary cause is an excessive intake of calories over the daily expenditure of energy. Unfortunately, the nature of modern life has influenced individuals to

(c) The Author(s) 2021. This article is licensed under a Creative Commons Attribution 4.0 International License, which permits use, sharing, adaptation, distribution and reproduction in any medium or format, as long as you give appropriate credit to the original author(s) and the source, provide a link to the Creative Commons licence, and indicate if changes were made. The images or other third party material in this article are included in the article's Creative Commons licence, unless indicated otherwise in a credit line to the material. If material is not included in the article's Creative Commons licence and your intended use is not permitted by statutory regulation or exceeds the permitted use, you will need to obtain permission directly from the copyright holder. To view a copy of this licence, visit http://creativecommons.org/licenses/by/4.0/. 
be less physically active, and there has been a dramatic increase in fast food restaurants. Since fast food is cheap and readily available, individuals often make it their first choice. Although it has a rich and satisfying taste, fast food is high in calories and contains excessive fat and sugar. Studies have shown that frequent visits to fast food restaurants affect weight gain [5, 25, 29, 30].

The Organization for Economic Co-operation and Development (OECD) published a report [26] identifying a significant increase in obesity rates in countries around the world. The United States was at the top of the OECD list, where obesity increased from $14 \%$ to approximately $34 \%$, which is about one third of the population. Also, the Ministry of Health in Saudi Arabia conducted a statistical survey on obesity in 2013 [22]. These statistics showed that obesity reached 24\% among males and 34\% among females in Saudi Arabia. In recent years, more chronic diseases, such as high blood pressure, diabetes, heart attack, and cancer, have spread around the world. One primary factor to being susceptible to these diseases is obesity $[7,11,23,27]$. As a result, obesity plays a vital role in reducing the average life of individuals.

It is highly likely that social interaction influences individual's habits. Researchers have specifically been examining the relationship between peer pressure and obesity. A study conducted on European children [16] reported a link between peer influences and obesity, with the largest effects on children in Spain, Italy, and Cyprus. The same connection was found in [24] for Chinese children and adolescents. One of the first studies to measure peer pressure found that adolescents with higher BMIs have a significant impact on their peers and that females are influenced more by their peers than males [32]. Similar evidence stated in [21] supports the claim that peer pressure significantly impacts the weight of adolescent females more than that of adolescent males. In addition, they found that same-gender peers have a stronger influence among females. Peer pressure in relation to weight-related behavior has also been investigated, especially in terms of how peer pressure among adolescents affects the frequency of eating fast food [4]. The findings in [15] showed a relation between fast food consumption among adolescents and their peers. However, there is minimal difference whether the effect of peers is used or ignored as a factor in the statistical analysis. Along with reducing the intake of calories, physical activity is an essential way to lose excess weight $[8,13,33$ ]. Hankinson et al. [17] conducted a longitudinal study over 20 years to evaluate the impact of high levels of physical activity on BMI and waist circumference. They found that young adults who maintained high levels of physical activity (at least 30 minutes a day) throughout their lives gained fewer kilograms during the transition to middle age. Similarly, Donnelly et al. [10] studied the effect of exercise on sedentary, overweight, and obese young adults. Without any change in their diet, a program consisting of 16 months of exercise produced a significant reduction in BMI for males and prevented weight gain in females.

Mathematical models have been used to study and analyze many social phenomena and epidemic diseases. They are capable of predicting global dynamic behavior and shedding light on factors with the most significant influence. Lately, researchers have employed models to investigate the role that fast food plays in relation to obesity. In 2004, Evangelista et al. [14] studied the role of peer pressure in fast food consumption, as well as its effect on individual weight in the United States. They found that a reduction in peer pressure to eat fast food could reduce obesity rates. Jodar et al. [20] developed an obesity model for children 3 to 5 years old, and they studied the effect of the bakery, fried meals, 
and soft drinks (BFS) on childhood obesity in the Spanish region of Valencia. They presented numerical simulations showing an increasing trend in childhood obesity from the previous years. Arenas et al. [6] extended the same model to the whole population, but with a non-autonomous term. They modified the transmission rate due to social pressure on BFS consumption as a time interval parameter. They obtained sufficient conditions to guarantee the existence of a periodic positive solution. Finally, Santonga et al. [31] investigated the effect of public health campaigns on reducing excess weight in Valencia. Using the numerical simulations, they concluded that preventive health campaigns to stop people from increasing their food intake were more effective than treatment; however, the combination of both was more efficient.

Most previous studies have examined the obesity models numerically due to their complexity. However, qualitative analysis of mathematical models $[9,12,18,28]$ presents more general results and allows for better understanding of the critical parameters that play a significant role in the dynamics of the models, see for example $[1-3,19]$.

In this work, we investigate the proposed mathematical models using both qualitative and numerical analysis. We build two models: model I studies the effect of peer pressure on obesity and fast food consumption; model II is an extension to model I, where workout factor is included. In both models, we find the equilibrium points and their existing conditions in relation to threshold numbers. Also, we explore the local and global conditions for the stability of equilibria using the linearization method and Lyapunov functions. Furthermore, we illustrate numerical simulations to support the qualitative results as well as analyzing the critical parameters of the models.

The outline of the paper is organized as follows. In Sect. 2, we present statistical analysis, which was carried out by the authors, regarding the peer pressure effects on fast food consumption and the impact of physical activity on obesity. In Sect. 3, we formulate model I by demonstrating the transmission mechanism, and then we investigate the model qualitatively and numerically. In Sect. 4, we add the workout factor to model I and examine model II by analyzing the role of exercise in reducing weight. Finally, we give a brief conclusion in Sect. 5.

\section{Statistical survey}

A cross-sectional online survey was carried out in Jeddah, Saudi Arabia from 26 April to 28 July 2016. Our aim was to explore the extent to which peer pressure influences individuals to eat at fast food restaurants in Jeddah. Also, we investigated the impact of consuming fast food meals and the role of physical activity on the weight of individuals.

Participants were recruited through technological social networks. The survey consisted of an online questionnaire that participants were asked to complete. The number of participants who completed the questionnaire was $n=1229$. The questionnaire was divided into two sections. The first section contained demographic questions about age, gender, BMI, and living area. The second section requested the participant' behavior towards consuming fast food and engaging in physical activity.

We analyzed the data obtained from the study by using SPSS (descriptive analysis) and Excel. The results are presented in this section.

The age distribution of the participants was 138 adolescents (11\%), 838 young adults (68\%), 175 middle-aged adults (14\%), and 78 older adults (6\%). Thus, the majority of the participants were young adults. Also, most of the participants, at a total of 1008 (82\%), 
Table 1 Demographic data of participants

\begin{tabular}{|c|c|c|c|c|c|}
\hline & & Frequency & Percent & Valid Percent & $\begin{array}{l}\text { Cumulative } \\
\text { Percent }\end{array}$ \\
\hline \multirow[t]{7}{*}{ Age } & $12-15$ years & 24 & 2 & 2 & 2 \\
\hline & $16-18$ years & 114 & 9.3 & 9.3 & 11.2 \\
\hline & 19-29 years & 542 & 44.1 & 44.1 & 55.3 \\
\hline & 30-39 years & 296 & 24.1 & 24.1 & 79.4 \\
\hline & 40-49 years & 175 & 14.2 & 14.2 & 93.7 \\
\hline & $50-60$ years & 69 & 5.6 & 5.6 & 99.3 \\
\hline & $>60$ years & 9 & 0.7 & 0.7 & 100 \\
\hline \multirow[t]{2}{*}{ Gender } & Male & 221 & 18 & 18 & 18 \\
\hline & Female & 1008 & 82 & 82 & 100 \\
\hline \multirow[t]{4}{*}{ BMI classification } & Underweight & 97 & 7.9 & 7.9 & 7.9 \\
\hline & Normal weight & 501 & 40.8 & 40.8 & 48.7 \\
\hline & Overweight & 354 & 28.8 & 28.8 & 77.5 \\
\hline & Obese & 277 & 22.5 & 22.5 & 100 \\
\hline \multirow[t]{4}{*}{ Living Area } & North of Jeddah & 551 & 44.8 & 44.8 & 44.8 \\
\hline & South east of Jeddah & 213 & 17.3 & 17.3 & 62.2 \\
\hline & South west of Jeddah & 172 & 14 & 14 & 76.2 \\
\hline & Center of Jeddah & 293 & 23.8 & 23.8 & 100 \\
\hline
\end{tabular}

were female. The participants were classified according to their weight using BMI as follows: 501 individuals (41\%) were normal-weight, 354 individuals (29\%) were overweight, 277 individuals (23\%) were obese, and 97 individuals (8\%) were underweight. In terms of the living areas of the participants, the majority (45\%) lived in north Jeddah, followed by $24 \%$ in the center of Jeddah, $17 \%$ in the southeast of Jeddah, and $14 \%$ in the southwest of Jeddah (see Table 1).

The investigation of the behavior of participants regarding fast food consumption revealed that most of them (86\%) ate fast food meals and the majority (93\%) had been eating at fast food restaurants for more than one year. The frequency of consumption varied as follows: 378 (36\%) ate fast food meals 1-2 times a month; 301 (29\%) ate fast food meals once a week; 292 (28\%) ate fast food meals 2 to 3 times a week, and only (8\%) ate fast food meals four or more times a week. Additionally, more than half of the participants (64\%) felt that consuming fast food meals increased their weight. In terms of the main objective that the study aimed to investigate, we found that 711 participants (58\%) reported that peer pressure from family and friends was one of the reasons they ate fast food meals. In terms of physical activity, almost half of the participants (45\%) performed exercises, with most of them (38\%) spending 2 to 3 hours a week working out. Furthermore, $65 \%$ of the participants felt that physical activity was effective in reducing their weight (see Table 2).

The analysis in Table 3 shows that $85 \%$ of overweight and $88 \%$ of obese participants consumed fast food. Also, $66 \%$ of obese and $65 \%$ of overweight participants ate fast food meals at least once a week. This suggests that there is a strong relationship between weight gain and fast food. In addition, we found that peer pressure was an influential factor that drove $70 \%$ of obese and $71 \%$ of overweight participants to consume fast food. As for physical activity, $56 \%$ of overweight participants who consumed fast food also worked out, compared to $37 \%$ of obese participants. 
Table 2 Characteristic of fast food consumption and behavior among participants

\begin{tabular}{llcccc}
\hline & & Frequency & Percent & Valid Percent & $\begin{array}{c}\text { Cumulative } \\
\text { Percent }\end{array}$ \\
\hline Eat fast food & Yes & 1054 & 85.8 & 85.8 & 85.8 \\
& No & 175 & 14.2 & 14.2 & 100 \\
I have been eating fast food since... & $1-3$ months & 32 & 2.6 & 3 & 3 \\
& $4-6$ months & 20 & 1.6 & 1.9 & 4.9 \\
& $7-9$ months & 11 & 0.9 & 1 & 6 \\
& $10-12$ months & 13 & 1.1 & 1.2 & 7.2 \\
Number of consuming fast food & more than a year & 978 & 79.6 & 92.8 & 100 \\
& $1-2$ times a month & 378 & 30.8 & 35.9 & 35.9 \\
& once a week & 301 & 24.5 & 28.6 & 64.4 \\
& 2-3 times a week & 292 & 23.8 & 27.7 & 92.1 \\
Do you eat fast food because & 2-4 times a week & 83 & 6.8 & 7.9 & 100 \\
of peer-pressure? & Yes & 711 & 57.9 & 57.9 & 57.9 \\
Do you feel you gain weight when & No & 518 & 42.1 & 42.1 & 100 \\
eating fast food? & Yes & 670 & 54.5 & 63.6 & 63.6 \\
Do you workout? & No & 384 & 31.2 & 36.4 & 100 \\
& Yes & 549 & 44.7 & 44.7 & 44.7 \\
How many hours do you workout? & No & 680 & 55.3 & 55.3 & 100 \\
& One a hour a week & 155 & 12.6 & 28.2 & 28.2 \\
& 2-3 hours a week & 209 & 17 & 38.1 & 66.3 \\
& $4-5$ hours a week & 126 & 10.3 & 23 & 89.3 \\
Do you feel you lose weight & 6-7 hours a week & 59 & 4.8 & 10.7 & 100 \\
when exercising? & Yes & 358 & 29.1 & 65.2 & 65.2 \\
\hline & No & 191 & 15.5 & 34.8 & 100 \\
\hline
\end{tabular}

Table 3 Distribution of overweight / obese behavior of fast food consumption

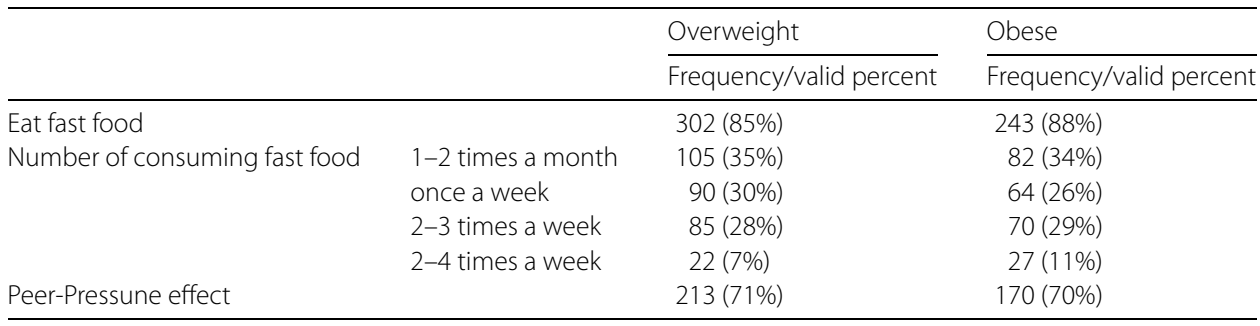

\section{Model I: peer pressure effect}

\subsection{Formulation of the model}

We build a mathematical model to study how peer pressure may affect the temptation that individuals face to eat at fast food restaurants and consequently gain weight. We consider a homogeneously mixed constant population at time $t$ denoted by $P(t)$. The population is divided into four separate classes of individuals: normal-weight individuals, $N(t)$; overweight individuals who eat fast food meals, $S(t)$; obese individuals who eat fast food meals, $O(t)$; and quitters who no longer eat fast food meals, $Q(t)$. We assume that normal-weight individuals enter compartment $S$ with rate $\beta$, as a result of peer pressure from overweight individuals who eat at fast food restaurants frequently. Also, overweight individuals enter compartment $O$ with rate $\gamma$ due to peer pressure from obese individuals who eat at fast food restaurants normally. On the other hand, quitters who no longer eat fast food may have positively influenced overweight and obese individuals by convincing them to refrain from consuming fast food to reduce their weight and susceptibility to harmful diseases. Accordingly, overweight and obese individuals move to compartment $Q$ with rates $\varepsilon_{1}$ and 


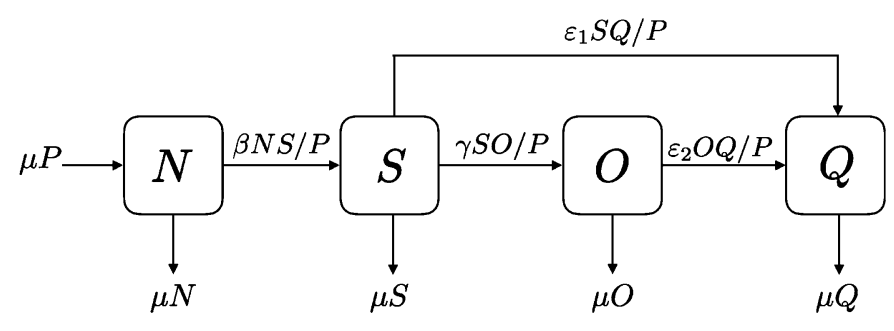

Figure 1 The dynamics of model I

$\varepsilon_{2}$, respectively. Finally, individuals enter and exit the model with rate $\mu$. The dynamics of the model are represented in Fig. 1, where all parameters belong to the interval $(0,1]$.

Based on the above assumptions, the model is governed by the following nonlinear system of ordinary differential equations:

$$
\begin{aligned}
& N^{\prime}(t)=\mu P-\beta N\left(\frac{S}{P}\right)-\mu N \\
& S^{\prime}(t)=\beta N\left(\frac{S}{P}\right)-\gamma S\left(\frac{O}{P}\right)-\varepsilon_{1} S\left(\frac{Q}{P}\right)-\mu S \\
& O^{\prime}(t)=\gamma S\left(\frac{O}{P}\right)-\varepsilon_{2} O\left(\frac{Q}{P}\right)-\mu O \\
& Q^{\prime}(t)=\varepsilon_{1} S\left(\frac{Q}{P}\right)+\varepsilon_{2} O\left(\frac{Q}{P}\right)-\mu Q
\end{aligned}
$$

where $P(t)=N(t)+S(t)+O(t)+Q(t)$. Note that $P^{\prime}(t)=0$, which means that the total population is constant. By rescaling the system with the following substitutions: $x=\frac{N}{P}, y=$ $\frac{S}{P}, z=\frac{O}{P}, q=\frac{Q}{P}$, we get

$$
\begin{aligned}
& x^{\prime}=\mu-\beta x y-\mu x, \\
& y^{\prime}=\beta x y-\gamma y z-\varepsilon_{1} y q-\mu y, \\
& z^{\prime}=\gamma y z-\varepsilon_{2} z q-\mu z, \\
& q^{\prime}=\varepsilon_{1} y q+\varepsilon_{2} z q-\mu q,
\end{aligned}
$$

with $x+y+z+q=1$. We consider the domain of the system to be

$$
\Gamma=\{(x, y, z, q): x+y+z+q \leq 1, x>0, y \geq 0, z \geq 0, q \geq 0\} .
$$

It can be easily proved that $\Gamma$ is a positively invariant set, i.e., every solution of system (2) starting with initial conditions in $\Gamma$ remains there for all $t>0$.

\subsection{Equilibrium points and basic reproduction number}

The free equilibrium point of system (2) is $E_{1,0}=(1,0,0,0)$, which represents a community with normal-weight individuals. Following epidemiological models, we obtain the basic reproduction number $R_{1,0}$ of the model by using the free equilibrium point and utilizing the method of next generation matrix [9]. In our model, $R_{1,0}$ represents the number of 
secondary cases of normal-weight individuals who start to gain weight due to consuming fast food meals under peer pressure from a single overweight individual who eats fast food frequently. Now, we define $F$ and $V$ as follows:

$$
F=\left[\begin{array}{c}
\beta x y \\
\gamma y z
\end{array}\right], \quad V=\left[\begin{array}{c}
\gamma y z+\varepsilon_{1} y q+\mu y \\
\varepsilon_{2} z q+\mu z
\end{array}\right] .
$$

The Jacobian matrices of $F$ and $V$ at the free equilibrium point $E_{1,0}$ are

$$
J\left(F\left(E_{1,0}\right)\right)=\left[\begin{array}{cc}
\beta & 0 \\
0 & 0
\end{array}\right], \quad J\left(V\left(E_{1,0}\right)\right)=\left[\begin{array}{cc}
\mu & 0 \\
0 & \mu
\end{array}\right] .
$$

Thus, the next generation matrix is

$$
J\left(F\left(E_{1,0}\right)\right) J\left(V\left(E_{1,0}\right)\right)^{-1}=\left[\begin{array}{cc}
\frac{\beta}{\mu} & 0 \\
0 & 0
\end{array}\right] .
$$

Hence, the basic reproduction number of the system is the spectral radius of matrix $J\left(F\left(E_{1,0}\right)\right) J\left(V\left(E_{1,0}\right)\right)^{-1}$, that is, $R_{1,0}=\frac{\beta}{\mu}$.

To find the equilibrium points of system (2), we solve the equations: $x^{\prime}=0, y^{\prime}=0, z^{\prime}=0$, and $q^{\prime}=0$, hence, the equilibrium points of the model are:

1. Overweight equilibrium $E_{1,1}=\left(\frac{\mu}{\beta}, 1-\frac{\mu}{\beta}, 0,0\right)$, which exists if $1-\frac{\mu}{\beta}>0$, i.e., if $R_{1,0}>1$.

2. Free quitters equilibrium $E_{1,2}=\left(\frac{\gamma}{\beta+\gamma}, \frac{\mu}{\gamma}, \frac{\beta}{\beta+\gamma}-\frac{\mu}{\gamma}, 0\right)$, which exists if $R_{1,1}=\frac{\beta \gamma}{\mu(\beta+\gamma)}>1$.

3. Free obese equilibrium $E_{1,3}=\left(\frac{\varepsilon_{1}}{\beta+\varepsilon_{1}}, \frac{\mu}{\varepsilon_{1}}, 0, \frac{\beta}{\beta+\varepsilon_{1}}-\frac{\mu}{\varepsilon_{1}}\right)$, which exists if $R_{1,2}=\frac{\beta \varepsilon_{1}}{\mu\left(\beta+\varepsilon_{1}\right)}>1$.

4. Positive equilibrium $E_{1,4}=\left(x_{1,4}, y_{1,4}, z_{1,4}, q_{1,4}\right)$, where $x_{1,4}=\left(\frac{\mu}{\beta y_{1,4}+\mu}\right), y_{1,4}=\left(\frac{\epsilon_{2}}{\gamma+\epsilon_{2}-\epsilon_{1}}\right)-$ $\left(\frac{\mu}{\beta}\right), z_{1,4}=\left(\frac{\mu+\epsilon_{1} y_{1,4}}{\epsilon_{2}}\right), q_{1,4}=\left(\frac{\gamma y_{1,4}-\mu}{\epsilon_{2}}\right)$. The positive equilibrium point exists if $y_{1,4}, z_{1,4}, q_{1,4}>0$. Set $R_{1,3}=\frac{\gamma+\varepsilon_{2}-\varepsilon_{1}}{\varepsilon_{2}}$, then $y_{1,4}>0$ if $R_{1,3}>0$ and $R_{1,0}>R_{1,3} ; z_{1,4}>0$ if $R_{1,3}>R_{1,2}$; and $q_{1,4}>0$ if $R_{1,1}>R_{1,3}$. Note that $\gamma<\beta+\gamma \Rightarrow \frac{\gamma}{\beta+\gamma}<1 \Rightarrow \frac{\beta \gamma}{\mu(\beta+\gamma)}<\frac{\beta}{\mu} \Rightarrow R_{1,1}<R_{1,0}$. Thus, $E_{1,4}$ exists if $0<R_{1,2}<R_{1,3}<R_{1,1}<R_{1,0}$.

Theorem 1 System (2) has five equilibria: free equilibrium point $E_{1,0}$ which exists always; overweight equilibrium point $E_{1,1}$ which exists if $R_{1,0}>1$; free quitters equilibrium point $E_{1,2}$ which exists if $R_{1,1}>1$; free obese equilibrium point $E_{1,3}$ which exists if $R_{1,2}>1$; and positive equilibrium point $E_{1,4}$ exists if $0<R_{1,2}<R_{1,3}<R_{1,1}<R_{1,0}$.

\subsection{Stability analysis}

We present in this section the study of local and global stability for the equilibrium points using the linearization method and Lyapunov function [14, 19, 24].

Local stability.

Theorem 2 (Local stability of $E_{1,0}$ ) The free equilibrium point $E_{1,0}$ of system (2) is locally asymptotically stable if $R_{1,0}<1$, locally stable if $R_{1,0}=1$, and unstable if $R_{1,0}>1$. 
Proof By using the linearization method [31], the Jacobian matrix of system (2) at the free equilibrium $E_{1,0}=(1,0,0,0)$ is given by

$$
J\left(E_{1,0}\right)=\left[\begin{array}{cccc}
-\mu & -\beta & 0 & 0 \\
0 & \beta-\mu & 0 & 0 \\
0 & 0 & -\mu & 0 \\
0 & 0 & 0 & -\mu
\end{array}\right]
$$

Clearly, the eigenvalues of the Jacobian matrix are: $\lambda_{1,2,3}=-\mu<0$ and $\lambda_{4}=\beta-\mu$. If $\lambda_{4}=\beta-\mu<0$, i.e., $R_{1,0}<1$, then the free equilibrium point $E_{1,0}$ is locally asymptotically stable. If $\beta=\mu$, i.e., $R_{1,0}=1 \Rightarrow \lambda_{4}=0$, therefore $E_{1,0}$ is locally stable, and if $\beta-\mu>0$, i.e., $R_{1,0}>1 \Rightarrow \lambda_{4}>0$, hence $E_{1,0}$ is unstable.

Theorem 3 (Local stability of $E_{1,1}$ ) The overweight equilibrium point $E_{1,1}$ of system (2) is locally asymptotically stable if $R_{1,1}<1$ and $R_{1,2}<1$, locally stable if $R_{1,1}=1$ and $R_{1,2}=1$, and unstable if $R_{1,1}>1$ or $R_{1,2}>1$.

Proof The Jacobian matrix of system (2) at the overweight equilibrium $E_{1,1}=\left(\frac{\mu}{\beta}, 1-\frac{\mu}{\beta}, 0,0\right)$ is given by

$$
J\left(E_{1,1}\right)=\left[\begin{array}{cccc}
-\beta & -\mu & 0 & 0 \\
\beta-\mu & 0 & -\gamma+\frac{\gamma \mu}{\beta} & -\varepsilon_{1}+\frac{\varepsilon_{1} \mu}{\beta} \\
0 & 0 & \gamma-\frac{\gamma \mu}{\beta}-\mu & 0 \\
0 & 0 & 0 & \varepsilon_{1}-\frac{\varepsilon_{1} \mu}{\beta}-\mu
\end{array}\right]
$$

The eigenvalues of the Jacobian matrix are: $\lambda_{1}=\gamma-\frac{\mu \gamma}{\beta}-\mu<0$ if $R_{1,1}<1, \lambda_{2}=\varepsilon_{1}-$ $\frac{\varepsilon_{1} \mu}{\beta}-\mu<0$ if $R_{1,2}<1$, and $\lambda_{3,4}$ satisfy the characteristic equation $\lambda^{2}+a_{1} \lambda+a_{2}=0$, where $a_{1}=\beta$ and $a_{2}=\mu(\beta-\mu)$. Since the existence condition of the overweight equilibrium $E_{1,1}$ is $R_{1,0}>1$, therefore, $\beta>\mu$, thus, $a_{1}>0$ and $a_{2}>0$, consequently, $\lambda_{3,4}$ have negative values. Therefore, by Routh-Hurwitz criteria, the overweight equilibrium $E_{1,1}$ is locally asymptotically stable when $R_{1,1}<1$ and $R_{1,2}<1$. If $R_{1,1}=1$ and $R_{1,2}=1$, then $\lambda_{1}=0$ and $\lambda_{2}=0$, So, $E_{1,1}$ is locally stable. In case $R_{1,1}>1$ or $R_{1,2}>1$, either $\lambda_{1}>0$ or $\lambda_{2}>0$. Thus, the overweight equilibrium $E_{1,1}$ is unstable.

In the same manner, we can prove the local stability of $E_{1,2}$ and $E_{1,3}$. Therefore, we only state the following theorems.

Theorem 4 (Local stability of $E_{1,2}$ ) The free quitters equilibrium point $E_{1,2}$ of system (2) is locally asymptotically stable if $R_{1,1}<R_{1,3}$, locally stable if $R_{1,1}=R_{1,3}$, and unstable if $R_{1,1}>R_{1,3}$.

Theorem 5 (Local stability of $E_{1,3}$ ) The free obese equilibrium point $E_{1,3}$ of system (2) is locally asymptotically stable if $R_{1,2}>R_{1,3}$, locally stable if $R_{1,2}=R_{1,3}$, and unstable if $R_{1,2}<$ $R_{1,3}$.

\section{Global stability.}

Here, we introduce a Lyapunov function [23] to prove the global stability of the positive equilibrium point $E_{1,4}$ of system (2). 
Theorem 6 (Global stability of $E_{1,4}$ ) The positive equilibrium point $E_{1,4}$ of system (2) is globally stable in $\Gamma$.

Proof Consider the Lyapunov function:

$$
\begin{aligned}
& L=x_{1,4}\left(\frac{x}{x_{1,4}}-1-\ln \left(x x_{1,4}\right)\right)+y_{1,4}\left(\frac{y}{y_{1,4}}-1-\ln \left(y y_{1,4}\right)\right)+z_{1,4}\left(\frac{z}{z_{1,4}}-1-\ln \left(z z_{1,4}\right)\right) \\
& +q_{1,4}\left(\frac{q}{q_{1,4}}-1-\ln \left(q q_{1,4}\right)\right) \\
& L^{\prime}=\left(1-\frac{x_{1,4}}{x}\right) x^{\prime}+\left(1-\frac{y_{1,4}}{y}\right) y^{\prime}+\left(1-\frac{z_{1,4}}{z}\right) z^{\prime}+\left(1-\frac{q_{1,4}}{q}\right) q^{\prime} \\
& =\left[\left(1-\frac{x_{1,4}}{x}\right)(\mu-\beta x y-\mu x)\right]+\left[\left(1-\frac{y_{1,4}}{y}\right)\left(\beta x y-\gamma y z-\varepsilon_{1} y q-\mu y\right)\right] \\
& +\left[\left(1-\frac{z_{1,4}}{z}\right)\left(\gamma y z-\varepsilon_{2} z q-\mu z\right)\right]+\left[\left(1-\frac{q_{1,4}}{q}\right)\left(\varepsilon_{1} y q+\varepsilon_{2} z q-\mu q\right)\right] \\
& =\left[\left(1-\frac{x_{1,4}}{x}\right)\left(\beta x_{1,4} y_{1,4}+\mu x_{1,4}-\beta x y-\mu x\right)\right]+\left[\left(1-\frac{y_{1,4}}{y}\right)(\beta x y-\gamma y z\right. \\
& \left.\left.-\varepsilon_{1} y q-\left(\beta x_{1,4}-\gamma z_{1,4}-\varepsilon_{1} q_{1,4}\right) y\right)\right]+\left[\left(1-\frac{z_{1,4}}{z}\right)\left(\gamma y z-\varepsilon_{2} z q-\left(\gamma y_{1,4}-\varepsilon_{2} q_{1,4}\right) z\right)\right] \\
& +\left[\left(1-\frac{q_{1,4}}{q}\right)\left(\varepsilon_{1} y q+\varepsilon_{2} z q-\left(\varepsilon_{1} y_{1,4}+\varepsilon_{2} z_{1,4}\right) q\right)\right] \\
& =\left[\beta x_{1,4} y_{1,4}\left(1-\frac{x_{1,4}}{x}\right)\left(1-\frac{x y}{x_{1,4} y_{1,4}}\right)+\mu x_{1,4}\left(1-\frac{x_{1,4}}{x}\right)\left(1-\frac{x}{x_{1,4}}\right)\right] \\
& +\left[\beta x_{1,4} y_{1,4}\left(1-\frac{y_{1,4}}{y}\right)\left(\frac{x y}{x_{1,4} y_{1,4}}-\frac{y}{y_{1,4}}\right)+\gamma y_{1,4} z_{1,4}\left(1-\frac{y_{1,4}}{y}\right)\left(\frac{y}{y_{1,4}}-\frac{y z}{y_{1,4} z_{1,4}}\right)\right. \\
& \left.+\varepsilon_{1} y_{1,4} q_{1,4}\left(1-\frac{y_{1,4}}{y}\right)\left(\frac{y}{y_{1,4}}-\frac{y q}{y_{1,4} q_{1,4}}\right)\right] \\
& +\left[\gamma y_{1,4} z_{1,4}\left(1-\frac{z_{1,4}}{z}\right)\left(\frac{y z}{y_{1,4} z_{1,4}}-\frac{z}{z_{1,4}}\right)+\varepsilon_{2} z_{1,4} q_{1,4}\left(1-\frac{z_{1,4}}{z}\right)\left(\frac{z}{z_{1,4}}-\frac{z q}{z_{1,4} q_{1,4}}\right)\right] \\
& +\left[\varepsilon_{1} y_{1,4} q_{1,4}\left(1-\frac{q_{1,4}}{q}\right)\left(\frac{y q}{y_{1,4} q_{1,4}}-\frac{q}{q_{1,4}}\right)+\varepsilon_{2} z_{1,4} q_{1,4}\left(1-\frac{q_{1,4}}{q}\right)\left(\frac{z q}{z_{1,4} q_{1,4}}-\frac{q}{q_{1,4}}\right)\right] \\
& =\mu x_{1,4}\left(1-\frac{x_{1,4}}{x}\right)\left(1-\frac{x}{x_{1,4}}\right)+\left[\beta x_{1,4} y_{1,4}\left(1-\frac{x_{1,4}}{x}\right)\left(1-\frac{x y}{x_{1,4} y_{1,4}}\right)\right. \\
& \left.+\beta x_{1,4} y_{1,4}\left(1-\frac{y_{1,4}}{y}\right)\left(\frac{x y}{x_{1,4} y 1,4}-\frac{y}{y_{1,4}}\right)\right] \\
& +\left[\gamma y_{1,4} z_{1,4}\left(1-\frac{y_{1,4}}{y}\right)\left(\frac{y}{y_{1,4}}-\frac{y z}{y_{1,4} z_{1,4}}\right)+\gamma y_{1,4} z_{1,4}\left(1-\frac{z_{1,4}}{z}\right)\left(\frac{y z}{y_{1,4} z_{1,4}}-\frac{z}{z_{1,4}}\right)\right] \\
& +\left[\varepsilon_{1} y_{1,4} q_{1,4}\left(1-\frac{y_{1,4}}{y}\right)\left(\frac{y}{y_{1,4}}-\frac{y q}{y_{1,4} q_{1,4}}\right)+\varepsilon_{1} y_{1,4} q_{1,4}\left(1-\frac{q_{1,4}}{q}\right)\left(\frac{y q}{y_{1,4} q_{1,4}}-\frac{q}{q_{1,4}}\right)\right] \\
& +\left[\varepsilon_{2} z_{1,4} q_{1,4}\left(1-\frac{z_{1,4}}{z}\right)\left(\frac{z}{z_{1,4}}-\frac{z q}{z_{1,4} q_{1,4}}\right)+\varepsilon_{2} z_{1,4} q_{1,4}\left(1-\frac{q_{1,4}}{q}\right)\left(\frac{z q}{z_{1,4} q_{1,4}}-\frac{q}{q_{1,4}}\right)\right] \\
& =\mu x_{1,4}\left(1-\frac{x_{1,4}}{x}\right)\left(1-\frac{x}{x_{1,4}}\right)
\end{aligned}
$$




$$
\begin{aligned}
& +\beta x_{1,4} y_{1,4}\left[1-\frac{x y}{x_{1,4} y_{1,4}}-\frac{x_{1,4}}{x}+\frac{y}{y_{1,4}}+\frac{x y}{x_{1,4} y_{1,4}}-\frac{y}{y_{1,4}}-\frac{x}{x_{1,4}}+1\right] \\
& +\gamma y_{1,4} z_{1,4}\left[\frac{y}{y_{1,4}}-\frac{y z}{y_{1,4} z_{1,4}}-1+\frac{z}{z_{1,4}}+\frac{y z}{y_{1,4} z_{1,4}}-\frac{z}{z_{1,4}}-\frac{y}{y_{1,4}}+1\right] \\
& +\varepsilon_{1} y_{1,4} q_{1,4}\left[\frac{y}{y_{1,4}}-\frac{y q}{y_{1,4} q_{1,4}}-1+\frac{q}{q_{1,4}}+\frac{y q}{y_{1,4} q_{1,4}}-\frac{q}{q_{1,4}}-\frac{y}{y_{1,4}}+1\right] \\
& +\varepsilon_{2} z_{1,4} q_{1,4}\left[\frac{z}{z_{1,4}}-\frac{z q}{z_{1,4} q_{1,4}}-1\right. \\
& \left.+\frac{q}{q_{1,4}}+\frac{z q}{z_{1,4} q_{1,4}}-\frac{q}{q_{1,4}}-\frac{z}{z_{1,4}}+1\right] \\
& =\mu x_{1,4}\left(1-\frac{x_{1,4}}{x}\right)\left(1-\frac{x}{x_{1,4}}\right)+\frac{\beta y_{1,4}}{x}\left(2 x x_{1,4}-x_{1,4}^{2}-x^{2}\right) \\
& =-\left(\frac{\mu}{x}+\frac{\beta y_{1,4}}{x}\right)\left(x-x_{1,4}\right)^{2} .
\end{aligned}
$$

Since $L^{\prime}(x, y, z, q) \leq 0$, thus $E_{1,4}$ is globally stable in $\Gamma$.

\subsection{Numerical analysis}

In this section, we solve the model numerically and present simulations of the mathematical model to show its agreement with previous qualitative results. We considered the per capita peer pressure rate $\beta$ to be the product of two probabilities: the probability of a normal-weight individual meeting an overweight individual per week and the probability of them eating a fast food meal together. In the same manner, the remaining per capita peer pressure rates $\gamma, \varepsilon_{1}$, and $\varepsilon_{2}$ were estimated. The numerical simulations of the equilibrium points of model I are illustrated in Fig. 2 with initial conditions from the data in Tables 1 and 2 .

(i) Free equilibrium $E_{1,0}$. The equilibrium point satisfies the stability condition $R_{1,0}<1$. Therefore, we choose the following parameters: $\mu=0.02, \beta=0.01, \gamma=0.01, \varepsilon_{1}=0.01, \varepsilon_{2}=$ 0.01. The numerical simulations in Fig. 2(a) show that the long-term behavior of the solution curves of the system approaches the free equilibrium $E_{1,0}$. Hence the system is locally asymptotically stable about $E_{1,0}$ for the above set of parameters. In particular, we see the overweight, obese, and quitters compartments decrease to the value zero, and the normalweight compartment increases to the value 1 . This would mean, theoretically, that all individuals in the society eventually achieve normal-weight and refrain from consuming fast food.

(ii) Overweight equilibrium $E_{1,1}$. Figure 2(b) illustrates the changes in the four classes over time under the following parameter settings: $\mu=0.02, \beta=0.05, \gamma=0.01, \varepsilon_{1}=$ $0.01, \varepsilon_{2}=0.01$. The parameters satisfy the qualitative conditions of the overweight equilibrium: $R_{1,0}>1, R_{1,1}<1$, and $R_{1,2}<1$. The numerical simulations agree with the qualitative results since all solution curves of the system tend to the overweight equilibrium $E_{1,1}$. There is an increase in the number of normal-weight individuals as a result of the small number of overweight individuals. However, as time passes, the overweight individuals influence normal-weight individuals to eat fast food. With greater consumption of fast food, the number of normal-weights begins to decline to a balance level after reaching a certain peak. Consequently, the number of overweight individuals rises with increasing $\beta$ to an equilibrium level that is higher than the normal-weight level. In addition, the effect 


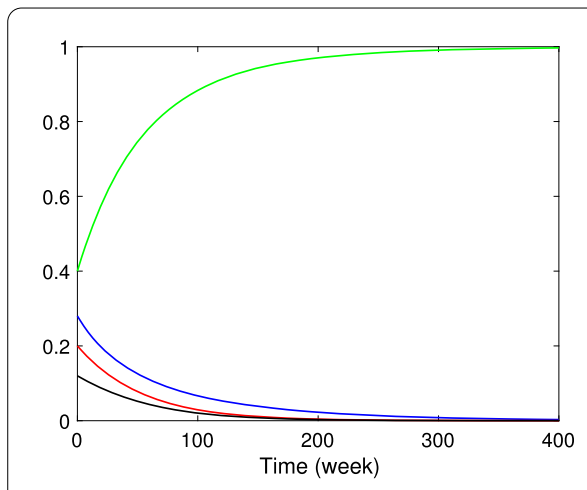

(a)

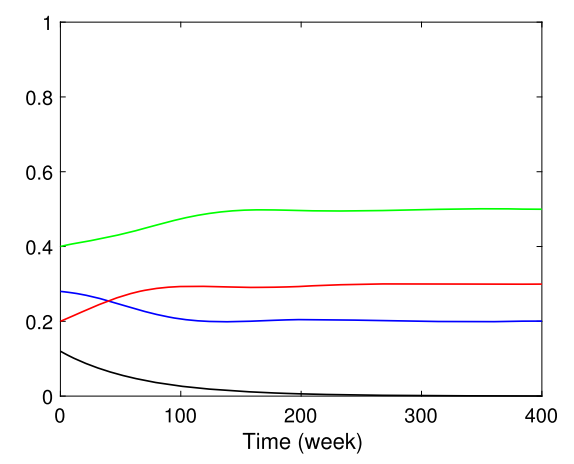

(c)

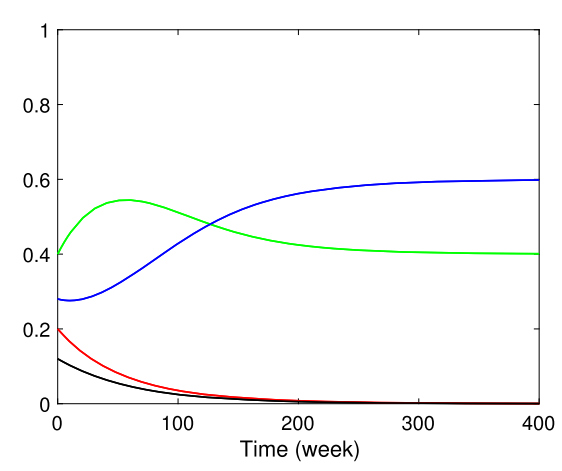

(b)

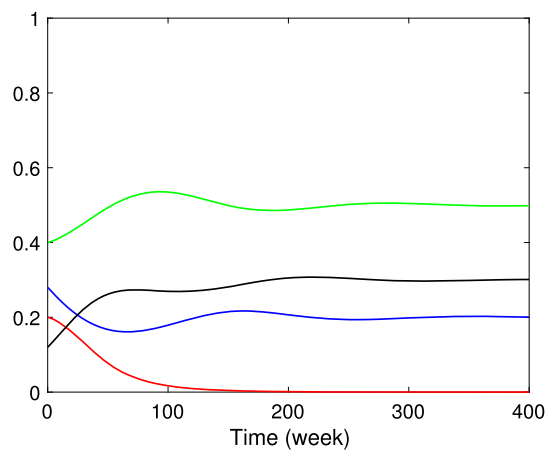

(d)

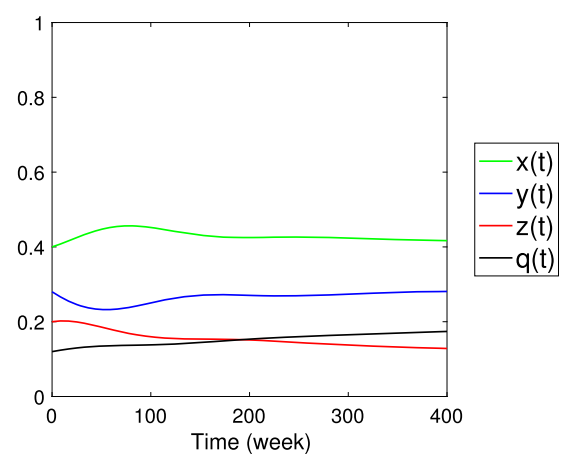

(e)

Figure 2 The dynamical behavior of system (2) with different sets of parameters. (a) Free equilibrium $E_{1,0}$, (b) Overweight equilibrium $E_{1,1}$, (c) Free quitter equilibrium $E_{1,2}$, (d) Free obese equilibrium $E_{1,3}$, (e) Positive equilibrium $E_{1,4}$

of obese and quitter individuals, in this case, is small in the society, and their numbers in time tend to zero.

(iii) Free quitters equilibrium $E_{1,2}$. In the same manner, Fig. 2(c) numerically indicates that the free quitters equilibrium $E_{1,2}$ is locally asymptotically stable. Under the qualitative conditions of $E_{1,2}: 1<R_{1,1}<R_{1,3}$, we set the parameters to be: $\mu=0.02, \beta=0.1, \gamma=$ $0.1, \varepsilon_{1}=0.01, \varepsilon_{2}=0.01$. The variation trend in the simulation is different from the previous cases. The number of obese individuals increases to a certain level as a result of their influence on overweight individuals to consume more fast food meals. Consequently, a decline in the number of overweight individuals leads to a rise in the number of normal-weight 


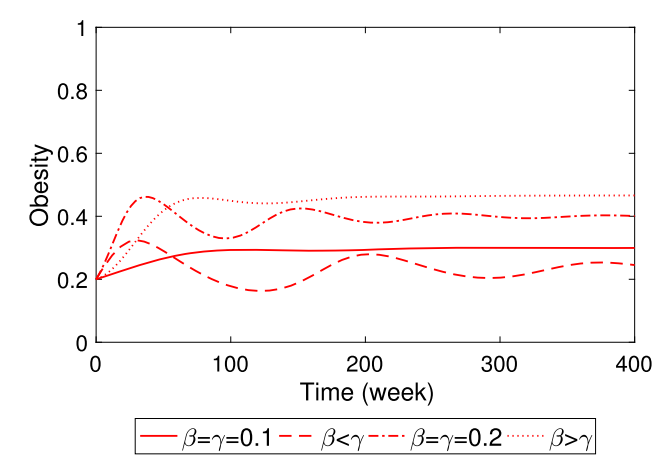

Figure 3 Obese compartment vs. time with varying $\beta$ and $\gamma$

individuals. This indicates that the impact of overweight individuals on normal-weight individuals over time decreases, since the rise of obese individuals made it easy for overweight individuals to find friends to engage in eating at fast food restaurants with them. The effect of quitters, in this case, is still small and their numbers in time tend to zero.

(iv) Free obese equilibrium $E_{1,3}$. The numerical simulations in Fig. 2(d) exhibit the local asymptotic stability of the free obese equilibrium $E_{1,3}$. We set the parameters to be: $\mu=0.02, \beta=0.1, \gamma=0.1, \varepsilon_{1}=0.1, \varepsilon_{2}=0.1$, which satisfy the conditions of $E_{1,3}: R_{1,2}>1$ and $R_{1,2}>R_{1,3}$. In this case, the simulation shows that the normal-weight and overweight classes at first behave in the same manner as in the previous case. However, as the number of quitters starts to rise, the number of overweight and obese individuals decreases below the number of quitters. This reflects the influence that quitters had on overweight and obese individuals to stop eating fast food meals; for this reason, the number of obese individuals eventually tends toward zero.

(v) Positive equilibrium $E_{1,4}$. In this last case, we set the parameters as follows: $\mu=$ $0.02, \beta=0.1, \gamma=0.1, \varepsilon_{1}=0.05, \varepsilon_{2}=0.05$ to satisfy the conditions of $E_{1,4}: 0<R_{1,2}<R_{1,3}<$ $R_{1,1}<R_{1,0}$. The simulations in Fig. 2(e) show that $E_{1,4}$ is locally asymptotically stable, thus, all classes exist. This is because the influence of quitters is not as low as in Fig. 2(c) and not as high as in Fig. 2(d).

\subsection{Parameter analysis}

The variation in the classes in model I is affected by the influential forces of peer pressure from overweight, obese, and quitter individuals. In this section, we examine the effect of peer pressure parameters, particularly on the obese class.

(i) Parameters $\beta, \gamma$. We investigate the effects on obesity as a result of the changes in the overweight and obese peer pressure rates. The numerical simulations in Fig. 3 demonstrate the time variation of obesity when the overweight peer pressure rate $\beta$ is compared with the obese peer pressure rate $\gamma$. Note that all other parameters are set to be the following values: $\mu=0.02, \varepsilon_{1}=0.01, \varepsilon_{2}=0.01$. The simulations show that obesity has higher values when $\beta>\gamma$ compared to when $\beta<\gamma$. In addition, the level of obesity is greater when $\beta=\gamma=0.2$ than when $\beta=\gamma=0.1$. Under these circumstances, we find that obesity is high when $\beta$ is large and vice versa. In other words, the comparison of the overweight and obese peer pressure rates indicates that the overweight peer pressure rate plays a more significant role in confining obesity. 


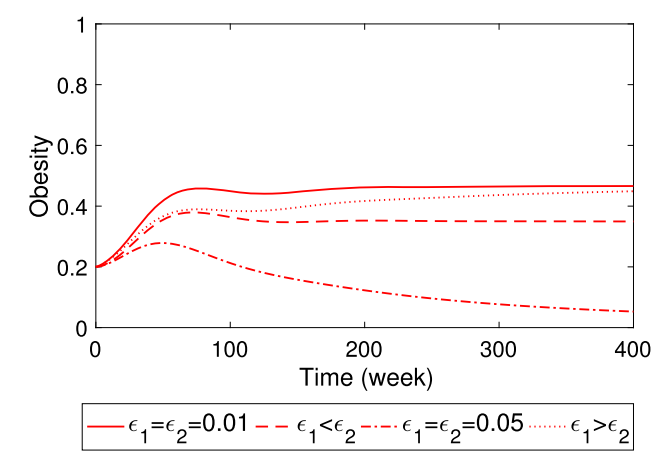

Figure 4 Obese compartment vs. time with varying $\varepsilon_{1}$ and $\varepsilon_{2}$

(ii) Parameters $\varepsilon_{1}, \varepsilon_{2}$. We explore how the levels of obesity are impacted by alterations to the peer pressure rate of quitter on overweight $\left(\varepsilon_{1}\right)$ and the pressure rate of quitter on obese $\left(\varepsilon_{2}\right)$. To begin, we set obesity at the highest level (from the previous case), that is, when $\beta=0.2$ and $\gamma=0.1$. The numerical simulations in Fig. 4 illustrate the time variation of obesity in four cases: $\varepsilon_{1}=\varepsilon_{2}=0.01, \varepsilon_{1}<\varepsilon_{2}, \varepsilon_{1}=\varepsilon_{2}=0.05$, and $\varepsilon_{1}>\varepsilon_{2}$. From these simulations, we find that the level of obesity declines when $\varepsilon_{1}<\varepsilon_{2}$. Furthermore, obesity decreases to a lower level when $\varepsilon_{1}=\varepsilon_{2}=0.05$. It appears as if the quitters' peer pressure rates had a major impact in helping to control the fast food eating habits of obese individuals.

In conclusion, the analysis designated two key parameters: $\beta$ and $\varepsilon_{2}$ as control strategies for obesity. The first strategy is to minimize the peer pressure from overweight individuals rather from obese individuals. The second strategy is to maximize the role of quitters in convincing obese individuals to stop consuming fast food meals.

\section{Model II: workout effect}

\subsection{Formulation of the model}

We modify model I to take into account the individual workout factor. We assume that exercising causes overweight individuals to enter compartment $N$ with rate $\alpha_{1}$ and obese individuals to move to compartment $S$ with rate $\alpha_{2}$. Individuals in class $Q$ may lose weight by avoiding fast food meals as well as performing exercises. After losing weight, some quitters may be susceptible to returning to the fast food life style and enter compartment $N$ with rate $\alpha_{3}$. Our aim is to determine the effect of exercise in reducing weight among overweight and obese individuals who frequently eat at fast food restaurants and to inspect the fallback effect of quitters on obesity. The dynamics of the model are presented in Fig. 5. Again, all parameters belong to the interval $(0,1]$.

The model is governed by the following nonlinear system:

$$
\begin{aligned}
& N^{\prime}(t)=\mu P-\beta N\left(\frac{S}{P}\right)+\alpha_{1} S+\alpha_{3} Q-\mu N, \\
& S^{\prime}(t)=\beta N\left(\frac{S}{P}\right)-\gamma S\left(\frac{O}{P}\right)-\varepsilon_{1} S\left(\frac{Q}{P}\right)-\alpha_{1} S+\alpha_{2} O-\mu S, \\
& O^{\prime}(t)=\gamma S\left(\frac{O}{P}\right)-\varepsilon_{2} O\left(\frac{Q}{P}\right)-\alpha_{2} O-\mu O,
\end{aligned}
$$




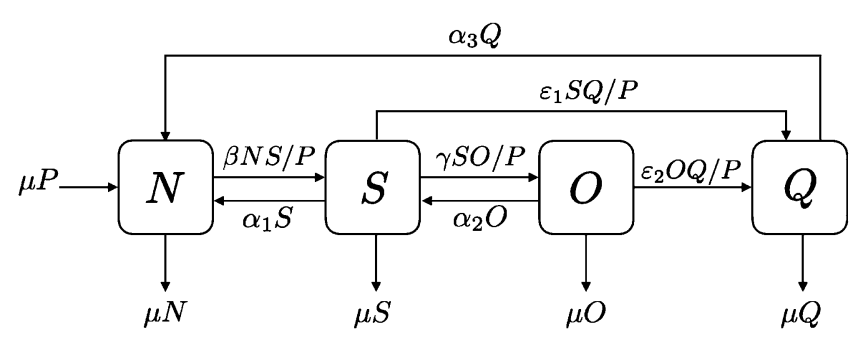

Figure 5 The dynamics of model II

$$
Q^{\prime}(t)=\varepsilon_{1} S\left(\frac{Q}{P}\right)+\varepsilon_{2} O\left(\frac{Q}{P}\right)-\alpha_{3} Q-\mu Q
$$

where $P(t)=N(t)+S(t)+O(t)+Q(t)$. Note that $P^{\prime}(t)=0$, The total population here is again constant. By rescaling the system with the same substitution as in model I: $x=\frac{N}{P}, y=\frac{S}{P}, z=$ $\frac{O}{P}, q=\frac{Q}{P}$, we get

$$
\begin{aligned}
& x^{\prime}=\mu-\beta x y+\alpha_{1} y+\alpha_{3} q-\mu x, \\
& y^{\prime}=\beta x y-\gamma y z-\varepsilon_{1} y q-\alpha_{1} y+\alpha_{2} z-\mu y, \\
& z^{\prime}=\gamma y z-\varepsilon_{2} z q-\alpha_{2} z-\mu z, \\
& q^{\prime}=\varepsilon_{1} y q+\varepsilon_{2} z q-\alpha_{3} q-\mu,
\end{aligned}
$$

with $x+y+z+q=1$. Consider the domain of the system to be

$$
\Gamma=\{(x, y, z, q): x+y+z+q \leq 1, x>0, y \geq 0, z \geq 0, q \geq 0\} .
$$

Again, $\Gamma$ is a positively invariant set.

\subsection{Equilibrium points and basic reproduction number}

The free equilibrium point of system (4) is $E_{2,0}=(1,0,0,0)$, that is, all the individuals in the society have normal-weight. By using the next generation matrix [9], we obtain the basic reproduction number $R_{2,0}$ of model II: $R_{2,0}=\frac{\beta}{\alpha_{1}+\mu}$. As in model I, $R_{2,0}$ represents the number of normal-weight individuals affected by the peer pressure of a single overweight individual to start gaining weight due to consuming fast food.

By letting the rates equal zero, i.e.; $x^{\prime}=0, y^{\prime}=0, z^{\prime}=0$, and $q^{\prime}=0$, we obtain the equilibrium points of system (4):

1. Overweight equilibrium $E_{2,1}=\left(\frac{\alpha_{1}+\mu}{\beta}, 1-\frac{\alpha_{1}+\mu}{\beta}, 0,0\right)$, which exists if $1-\frac{\alpha_{1}+\mu}{\beta}>0$, i.e., if $R_{2,0}>1$.

2. Free quitters equilibrium $E_{2,2}=\left(\frac{\alpha_{1}\left(\alpha_{2}+\mu\right)+\gamma \mu}{\beta\left(\alpha_{2}+\mu\right)+\gamma \mu}, \frac{\alpha_{2}+\mu}{\gamma},-\frac{\left(\alpha_{2}+\mu\right)\left(\alpha_{2} \beta+\alpha_{1} \gamma-\beta \gamma+\beta \mu+\gamma \mu\right)}{\gamma\left(\alpha_{2} \beta+\beta \mu+\gamma \mu\right)}, 0\right)$, which exists if $R_{2,1}=\frac{\beta \gamma}{\gamma\left(\alpha_{1}+\mu\right)+\beta\left(\alpha_{2}+\mu\right)}>1$.

3. Free obese equilibrium $E_{2,3}=\left(\frac{\varepsilon_{1}+\alpha_{1}-\alpha_{3}}{\beta+\varepsilon_{1}}, \frac{\alpha_{3}+\mu}{\varepsilon_{1}}, 0, \frac{\beta\left(\varepsilon_{1}+\alpha_{1}-\alpha_{3}\right)-\left(\alpha_{1}+\mu\right)\left(\beta+\varepsilon_{1}\right)}{\varepsilon_{1}\left(\varepsilon_{1}+\beta\right)}\right)$, which exists if $R_{2,2}=\frac{\beta \varepsilon_{1}}{\varepsilon_{1}\left(\alpha_{1}+\mu\right)+\beta\left(\alpha_{3}+\mu\right)}>1$.

4. Positive equilibrium $E_{2,4}=\left(x_{2,4}, y_{2,4}, z_{2,4}, q_{2,4}\right)$, where $x_{2,4}=\left(\frac{\varepsilon_{2}\left(\alpha_{1} y_{4}+\mu\right)+\alpha_{3}\left(\gamma y_{4}-\alpha_{2}-\mu\right)}{\varepsilon_{2}\left(\beta y_{4}+\mu\right)}\right)$, $z_{2,4}=\left(\frac{\mu+\alpha_{3}-\varepsilon_{1} y_{4}}{\varepsilon_{2}}\right), q_{2,4}=\left(\frac{\gamma y_{4}-\alpha_{2}-\mu}{\varepsilon_{2}}\right)$, and $y_{2,4}$ satisfy the characteristic equation $a y_{2,4}^{2}+b y_{2,4}+$ $c=0$, where $a=\beta\left(\gamma+\varepsilon_{2}-\varepsilon_{1}\right), b=\varepsilon_{2}\left(\alpha_{1}-\beta\right)+\alpha_{3}(\beta+\gamma)-\alpha_{2} \beta+\mu\left(\gamma+\varepsilon_{2}-\varepsilon_{1}\right), c=$ $-\alpha_{2}\left(\alpha_{3}+\mu\right) . E_{2.4}$ exists if $R_{2,3}=\frac{\gamma+\varepsilon_{2}}{\varepsilon_{1}}>1$ and $\frac{\alpha_{2}+\mu}{\gamma}<y_{2,4}<\frac{\alpha_{3}+\mu}{\varepsilon_{1}}$, where $y_{2.4}=\frac{-b+\sqrt{b^{2}-4 a c}}{2 a}$. 
Theorem 7 System (4) has five equilibrium points: free equilibrium point $E_{2,0}$ which exists always; overweight equilibrium point $E_{2,1}$ which exists if $R_{2,0}>1$; free quitters equilibrium point $E_{2,2}$ which exists if $R_{2,1}>1$; free obese equilibrium point $E_{2,3}$ which exists if $R_{2,2}>1$; and positive equilibrium point $E_{2,4}$ which exists if $R_{2,3}>1$ and $\frac{\alpha_{2}+\mu}{\gamma}<y_{2,4}<\frac{\alpha_{3}+\mu}{\varepsilon_{1}}$.

\subsection{Stability analysis}

In this section, we present a local stability analysis of all equilibrium points of system (4) using the linearization method [28] and Routh-Hurwitz criteria [12].

\section{Local stability.}

Theorem 8 (Local stability of $E_{2,0}$ ) The free equilibrium point $E_{2,0}$ of system (4) is locally asymptotically stable if $R_{2,0}<1$, locally stable if $R_{2,0}=1$, and unstable if $R_{2,0}>1$.

Proof By using the linearization method, the Jacobian matrix of system (4) at the free equilibrium $E_{2,0}=(1,0,0,0)$ is given by

$$
J\left(E_{2,0}\right)=\left[\begin{array}{cccc}
-\mu & \alpha_{1}-\beta & 0 & \alpha_{3} \\
0 & \beta-\alpha_{1}-\mu & \alpha_{2} & 0 \\
0 & 0 & -\alpha_{2}-\mu & 0 \\
0 & 0 & 0 & -\alpha_{3}-\mu
\end{array}\right]
$$

The eigenvalues of the Jacobian matrix are: $\lambda_{1}=-\mu, \lambda_{2}=-\alpha_{2}-\mu, \lambda_{3}=-\alpha_{3}-\mu$, and $\lambda_{4}=\beta-\alpha_{1}-\mu$. If $\lambda_{4}<0 \Rightarrow \beta<\alpha_{1}+\mu$, i.e., $R_{2,0}<1$, then the free equilibrium point $E_{2,0}$ is locally asymptotically stable. If $\lambda_{4}=0 \Rightarrow \beta=\alpha_{1}+\mu$, i.e., $R_{2,0}=1$, then the free equilibrium point $E_{2,0}$ is locally stable. And if $\lambda_{4}>0 \Rightarrow \beta>\alpha_{1}+\mu$, i.e., $R_{2,0}>1$, then the free equilibrium point $E_{2,0}$ is unstable.

Theorem 9 (Local stability of $\left.E_{2,1}\right)$ The overweight equilibrium point $E_{2,1}$ of system (4) is locally asymptotically stable if $R_{2,1}<1$ and $R_{2,2}<1$, locally stable if $R_{2,1}=1$ and $R_{2,2}=1$, and unstable if $R_{2,1}>1$ or $R_{2,2}>1$.

Proof The Jacobian matrix of system (4) at the overweight equilibrium $E_{2,1}=\left(\frac{\alpha_{1}+\mu}{\beta}, 1-\right.$ $\left.\frac{\alpha_{1}+\mu}{\beta}, 0,0\right)$ is given by

$$
J\left(E_{2,1}\right)=\left[\begin{array}{cccc}
\alpha_{1}-\beta & -\mu & 0 & \alpha_{3} \\
\beta-\alpha_{1}-\mu & 0 & -\gamma+\frac{\gamma\left(\alpha_{1}+\mu\right)}{\beta}+\alpha_{2} & -\varepsilon_{1}+\frac{\varepsilon_{1}\left(\alpha_{1}+\mu\right)}{\beta} \\
0 & 0 & \gamma-\frac{\gamma\left(\alpha_{1} \beta \mu\right)}{\beta}-\alpha_{2}-\mu & 0 \\
0 & 0 & 0 & \varepsilon_{1}-\frac{\varepsilon_{1}\left(\alpha_{1}+\mu\right)}{\beta}-\alpha_{3}-\mu
\end{array}\right]
$$

The eigenvalues of the Jacobian matrix are: $\lambda_{1}=\gamma-\frac{\gamma}{\beta}\left(\alpha_{1}+\mu\right)-\alpha_{2}-\mu<0$ if $R_{2,1}<1$, $\lambda_{2}=\varepsilon_{1}-\frac{\varepsilon_{1}}{\beta}\left(\alpha_{1}+\mu\right)-\alpha_{3}-\mu<0$ if $R_{2,2}<1$, and $\lambda_{3,4}$ satisfy the characteristic equation $\lambda^{2}+a_{1} \lambda+a_{2}=0$, where $a_{1}=\beta-\alpha_{1}$ and $a_{2}=\mu\left(\beta-\alpha_{1}-\mu\right)$. Since the existence condition of the overweight equilibrium $E_{2,1}$ is $R_{2,0}>1$, therefore, $\beta>\alpha_{1}+\mu \Rightarrow \beta>\alpha_{1}$ and $\beta>\mu$, thus, $a_{1}>0$ and $a_{2}>0$, consequently, $\lambda_{3,4}$ have negative values. Therefore, by Routh-Hurwitz criteria, the overweight equilibrium $E_{2,1}$ is locally asymptotically stable when $R_{2,1}<1$ and $R_{2,2}<1$. If $R_{2,1}=1$ and $R_{2,2}=1$, then $\lambda_{1}=0$ and $\lambda_{2}=0$, So, $E_{2,1}$ is locally stable. In case 
$R_{2,1}>1$ or $R_{2,2}>1$, then either $\lambda_{1}>0$ or $\lambda_{2}>0$. Thus, the overweight equilibrium $E_{2,1}$ is unstable.

In the same manner, we can prove the local stability of $E_{2,2}$ and $E_{2,3}$. Therefore, we state the following theorems.

Theorem 10 (Local stability of $E_{2,2}$ ) The free quitters equilibrium point $E_{2,2}$ of system (4) is locally asymptotically stable if $R_{2,4}<1$, locally stable if $R_{2,4}=1$, and unstable if $R_{2,4}>1$, where $R_{2,4}=\frac{\varepsilon_{1}\left(\alpha_{2}+\mu\right)}{\gamma\left(\alpha_{3}+\mu\right)}-\frac{\varepsilon_{2}\left(\alpha_{2}+\mu\right)\left(\alpha_{2} \beta+\alpha_{1} \gamma-\beta \gamma+\beta \mu+\gamma \mu\right)}{\gamma\left(\alpha_{3}+\mu\right)\left(\alpha_{2} \beta+\beta \mu+\gamma \mu\right)}$.

Theorem 11 (Local stability of $E_{2,3}$ ) The free obese equilibrium point $E_{2,3}$ of system (4) is locally asymptotically stable if $R_{2,5}<1$, locally stable if $R_{2,5}=1$, unstable if $R_{2,5}>1$, where $R_{2,5}=\frac{\left(\beta+\varepsilon_{1}\right)\left[\gamma\left(\alpha_{3}+\mu\right)+\varepsilon_{2}\left(\alpha_{1}+\mu\right)\right]+\alpha_{3} \beta \varepsilon_{2}}{\varepsilon_{1}\left(\alpha_{2}+\mu\right)\left(\beta+\varepsilon_{1}\right)+\beta \varepsilon_{2}\left(\alpha_{1}+\varepsilon_{1}\right)}$.

As for the positive equilibrium point $E_{2,4}$ of system (4), the eigenvalues of its Jacobian matrix satisfy the characteristic equation $\lambda^{4}+b_{1} \lambda^{3}+b_{2} \lambda^{2}+b_{3} \lambda+b_{4}=0$, where $b_{1}=\beta y_{2,4}-$ $\beta x_{2,4}+\gamma z_{2,4}+\varepsilon_{1} q_{2,4}+2 \mu+\alpha_{1}, b_{2}=\left(\mu+\beta y_{2,4}\right)\left(\alpha_{1}+\mu+\varepsilon_{1} q_{2,4}-\beta x_{2,4}+\gamma z_{2,4}\right)-\beta y_{2,4}\left(\alpha_{1}-\right.$ $\left.\beta x_{2,4}\right)-\gamma z_{2,4}\left(\alpha_{2}-\gamma y_{2,4}\right)+\varepsilon_{1}^{2} y_{2,4} q_{2,4}+\varepsilon_{2}^{2} z_{2,4} q_{2,4}, b_{3}=\left(\mu+\beta y_{2,4}\right)\left[\varepsilon_{1}^{2} y_{2,4} q_{2,4}+\varepsilon_{2}^{2} z_{2,4} q_{2,4}-\right.$ $\left.\gamma z_{2,4}\left(\alpha_{2}-\gamma y_{2,4}\right)\right]+\varepsilon_{2}^{2} z_{2,4} q_{2,4}\left(\alpha_{1}+\mu+\varepsilon_{1} q_{2,4}-\beta x_{2,4}+\gamma z_{2,4}\right)+\varepsilon_{1} \varepsilon_{2} z_{2,4} q_{2,4}\left(\alpha_{2}-\gamma y_{2,4}\right)-$ $\alpha_{3} \beta \varepsilon_{1} y_{2,4} q_{2,4}+\varepsilon_{1} \varepsilon_{2} \gamma y_{2,4} z_{2,4} q_{2,4}, b_{4}=\left(\mu+\beta y_{2,4}\right)\left[\varepsilon_{2}^{2} z_{2,4} q_{2,4}\left(\alpha_{1}+\mu+\varepsilon_{1} q_{2,4}-\beta x_{2,4}+\gamma z_{2,4}\right)+\right.$ $\left.\varepsilon_{1} \varepsilon_{2} z_{2,4} q_{2,4}\left(\alpha_{2}-\gamma y_{2,4}\right)+\varepsilon_{1} \varepsilon_{2} \gamma y_{2,4} z_{2,4} q_{2,4}\right]-\beta y_{2,4}\left[\varepsilon_{2}^{2} z_{2,4} q_{2,4}\left(\alpha_{1}-\beta x_{2,4}\right)+\alpha_{3} \gamma \varepsilon_{2} z_{2,4} q_{2,4}\right]$.

Based on Routh-Hurwitz criteria [12], $E_{2,4}$ is locally asymptotically stable if $b_{1}>0, b_{3}>$ $0, b_{4}>0$, and $b_{1} b_{2} b_{3}>b_{3}^{2}+b_{1}^{2} b_{4}$. We may state the following theorem.

Theorem 12 (Local stability of $E_{2,4}$ ) The positive equilibrium point $E_{2,4}$ of system (4) is locally asymptotically stable if $b_{1}>0, b_{3}>0, b_{4}>0$, and $b_{1} b_{2} b_{3}>b_{3}^{2}+b_{1}^{2} b_{4}$.

\subsection{Numerical analysis}

The numerical simulations of model II are presented in this section. The peer pressure parameters $\beta, \gamma, \varepsilon_{1}$, and $\varepsilon_{2}$ are defined in the same manner as model I. We set the following assumptions for the workout parameters. To begin, we suppose that an overweight individual who frequently eats at fast food restaurants gains $0.25 \mathrm{Kg}$ per week. Also, an overweight individual who walks at a fast pace for seven hours per week burns $3010 \mathrm{cal}$ which is approximately equal to $0.39 \mathrm{Kg}$. Therefore, an overweight individual who eats fast food meals and workout burns $0.14 \mathrm{Kg}$ per week. We assume that overweight individuals move to the normal-weight class if they burn $10 \mathrm{Kg}$. This process takes approximately 72 weeks. Thus, the overweight exercise rate $\alpha_{1}$ is equal to $1 / 72 \approx 0.0138$ per week. Likewise, we assume that an obese individual gains $0.25 \mathrm{Kg}$ from fast food meals but burns $0.44 \mathrm{Kg}$ per week from exercise. Therefore, the obese exercise rate $\alpha_{2}$ is equal to $1 / 105 \approx 0.0095$ per week, when assuming that obese individuals have to lose $20 \mathrm{Kg}$ to move to the overweight class. Furthermore, since the quitters class includes both overweight and obese individuals who do not eat fast food meals, we assume that the average calories burnt in this class from exercise is equal to $0.415 \mathrm{Kg}$ per week. Thus, the quitters exercise rate is equal to $1 / 48 \approx 0.0208$ per week, when assuming that individuals have to lose $20 \mathrm{Kg}$ to be in the normal-weight class. However, if we presume that $50 \%$ of the quitters, who have lost weight from exercising, enter a state of delusion and become vulnerable to fast food meals, then the rate $\alpha_{3}$ is equal to 0.0104 per week. From the previous assumptions, we set 


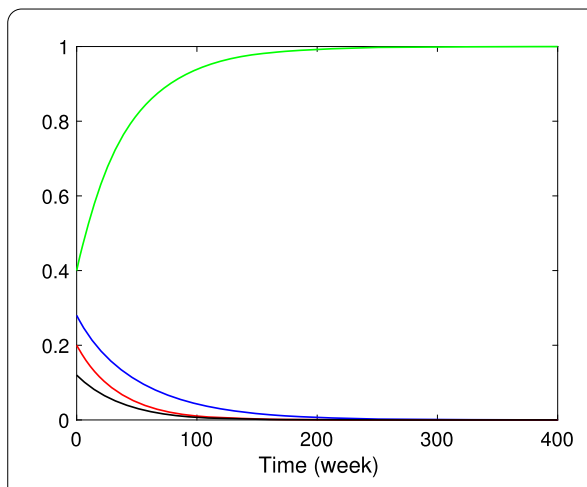

(a)

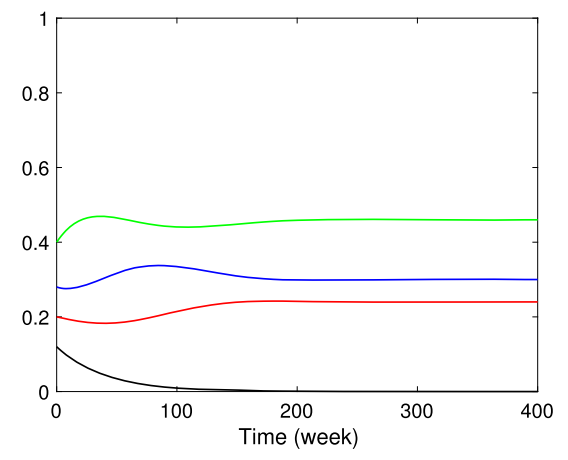

(c)

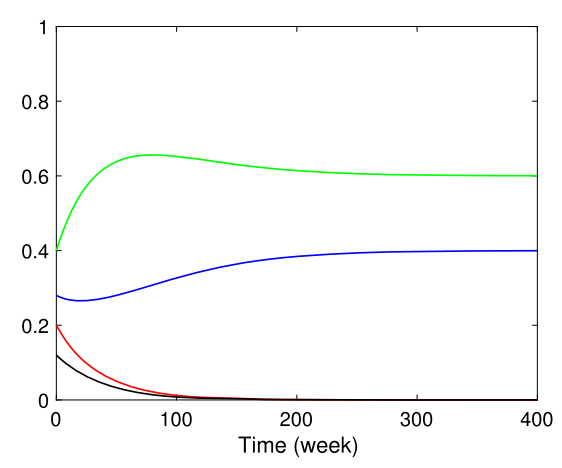

(b)

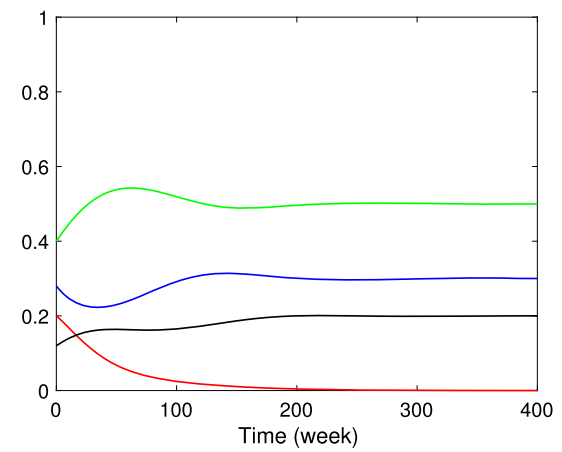

(d)

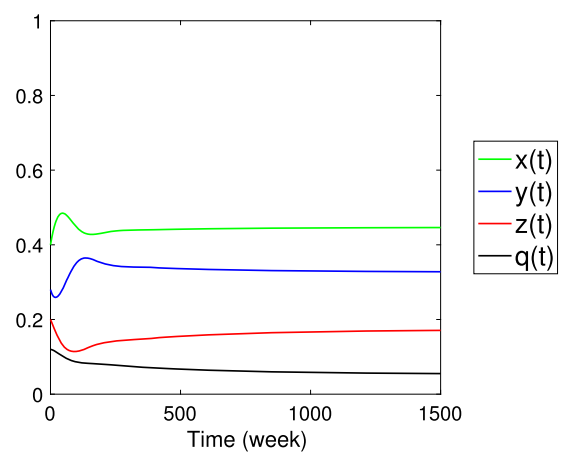

(e)

Figure 6 The dynamical behavior of system (4) with different sets of parameters. (a) Free equilibrium $E_{2,0}$, (b) Overweight equilibrium $E_{2,1}$, (c) Free quitter equilibrium $E_{2,2}$, (d) Free obese equilibrium $E_{2,3}$, (e) Positive equilibrium $E_{2,4}$

the workout parameters to be $\alpha_{1}=\alpha_{2}=\alpha_{3}=0.01$. On the other hand, the peer pressure parameters are chosen arbitrarily to verify the existence and local stability conditions. The initial conditions in the simulations are chosen from the data in Tables 1 and 2.

(i) Free equilibrium point $E_{2,0}$. The parameters were chosen to satisfy the existence and local stability conditions of $E_{2,0}$, that is, $R_{2,0}<1$. We set the parameters to be as follows: $\mu=$ $0.02, \beta=0.01, \gamma=0.01, \varepsilon_{1}=0.01, \varepsilon_{2}=0.01, \alpha_{1}=0.01, \alpha_{2}=0.01, \alpha_{3}=0.01$. The numerical simulations in Fig. 6(a) show that the long-term behavior of the solution curves of the system approaches $E_{2,0}$. In fact, we notice that the size of normal-weight class increases to its maximum value of one, while the overweight, obese, and quitter classes tend to 
zero. Thus, eventually, all individuals in the society have normal-weight. When comparing Figs. 6(a) and 2(a), the effect of exercise is demonstrated in lessening the time the system takes to reach the free equilibrium point.

(ii) Overweight equilibrium point $E_{2,1}$. The numerical simulations in Fig. 6(b) exhibit the local stability of the overweight equilibrium $E_{2,1}$ with the following choices of the parameters: $\mu=0.02, \beta=0.05, \gamma=0.01, \varepsilon_{1}=0.01, \varepsilon_{2}=0.01, \alpha_{1}=0.01, \alpha_{2}=0.01, \alpha_{3}=0.01$. The parameters satisfy the conditions of $E_{2,1}: R_{2,0}>1, R_{2,1}<1, R_{2,2}<1$. In this case, we note that the number of normal-weight and overweight individuals settles to a certain level in the long term, while the size of the obese and quitters groups tends to zero. When comparing the simulations in Fig. 6(b) with those in Fig. 2(b), we see that the size of the overweight class decreased due to the workout effect.

(iii) Free quitters equilibrium $E_{2,2}$. The chosen parameters to satisfy the existence and local stability conditions of $E_{2,2}$ are $\mu=0.02, \beta=0.1, \gamma=0.1, \varepsilon_{1}=0.01, \varepsilon_{2}=0.01, \alpha_{1}=$ $0.01, \alpha_{2}=0.01, \alpha_{3}=0.01$, where $R_{2,1}>1$ and $R_{2,4}<1$. As illustrated in Fig. 6(c), the solution curves tend to the free quitters equilibrium $E_{2,2}$. The number of obese individuals decreased as a result of physical activity, which increased the size of the overweight group. This is evident when comparing Fig. 6(c) with Fig. 2(c).

(iv) Free obese equilibrium $E_{2,3}$. In the same manner, Fig. 6(d) numerically shows that the free obese equilibrium $E_{2,3}$ is locally asymptotically stable. Under the qualitative conditions of $E_{2,3}: R_{2,2}>1$ and $R_{2,5}<1$, we set the parameters to be as follows: $\mu=0.02, \beta=$ $0.1, \gamma=0.1, \varepsilon_{1}=0.1, \varepsilon_{2}=0.1, \alpha_{1}=0.01, \alpha_{2}=0.01, \alpha_{3}=0.01$. Figure 6(d) shows that the population in the long term is divided into normal-weight, overweight, and quitter individuals, but no obese individuals. Furthermore, a comparison between Figs. 2(d) and 6(d) shows that the number of quitters has decreased due to the fallback of those who lost weight from exercise and returned to being vulnerable to fast food meals. Consequently, the size of the overweight class has increased owing to the effects of peer pressure.

(v) Positive equilibrium $E_{2,4}$. In the last case, the parameters satisfy the existence conditions of $E_{2,4}$. Therefore, we choose the following parameters: $\mu=0.02, \beta=0.1, \gamma=0.1, \varepsilon_{1}=$ $0.05, \varepsilon_{2}=0.05, \alpha_{1}=0.01, \alpha_{2}=0.01, \alpha_{3}=0.005$. The numerical simulation in Fig. 6(e) shows that the long-term behavior of the solution curves of the system approaches the positive equilibrium $E_{2,4}$. Hence the system is locally asymptotically stable at $E_{2,4}$ for the above set of parameters. When comparing Figs. 6(e) and 2(e), we see that the size of quitters has decreased as a result of exercise and being susceptible to returning to fast food meals. Moreover, the size of overweight and obese individuals has increased due to the effect of exercise and peer pressure.

\subsection{Parameter analysis}

In this section, we investigate the effects of exercise rates and peer pressure rates on obesity. For this purpose, we present numerical simulations for low and high workout rates, as opposed to low and high peer pressure rates. A society with only peer pressure rates and no workout rates is presented in model I, whereas model II undertakes to show the impact of both peer pressure and workouts. We set the workout parameters to be as follows: $\alpha_{1}=\alpha_{2}=\alpha_{3}=0.01$ (for low workout rates) and $\alpha_{1}=\alpha_{2}=\alpha_{3}=0.08$ (for high workout rates). The peer pressure parameters are set according to the following cases:

Case (1): We examine the case of low fast food peer pressure rates, that is, $\beta=\gamma=$ 0.05 , with low quitters' peer pressure rates of $\varepsilon_{1}=\varepsilon_{2}=0.01$. Under these considerations, 


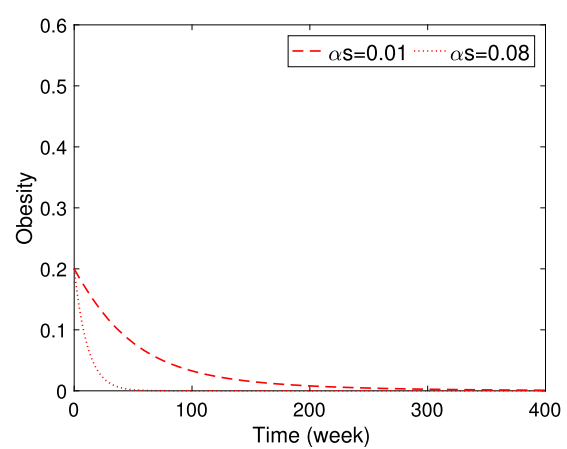

(a) Case (1)

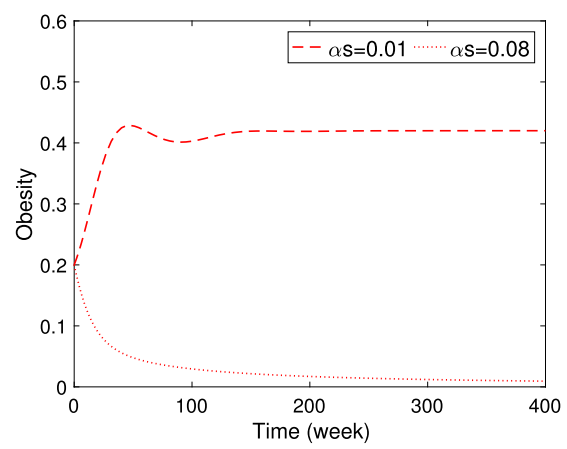

(c) Case (3)

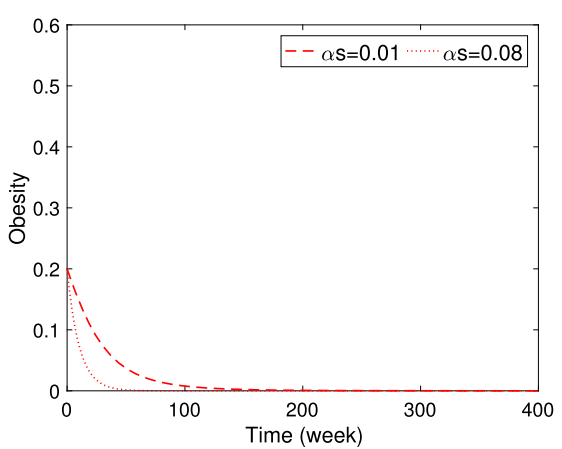

(b) Case (2)

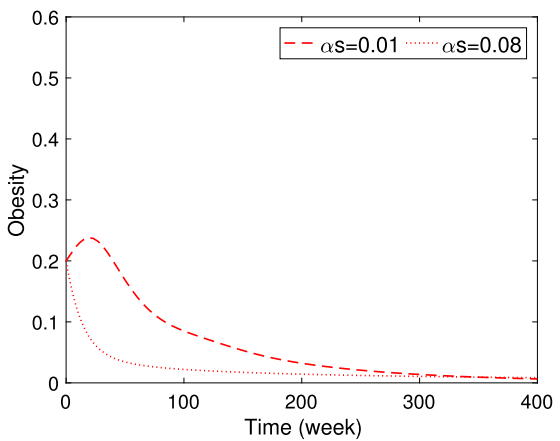

(d) Case (4)

Figure 7 Time variation of obesity for low and high workout raters with (a) low peer pressure rates, (b) low fast food pressure rates and high quitters peer pressure rates, (c) hight fast food peer pressure rates and low quitters peer pressure rates, and (d) high peer pressure rates

Fig. 7(a) demonstrates the time variation of obesity. The simulation shows that high workout rates fight obesity better than low workout rates. Also, high workout rates take less time to diminish obesity than low workout rates.

Case (2): We let the fast food peer pressure rates to be the same, that is, $\beta=\gamma=0.05$, but with high quitters' peer pressure rates of $\varepsilon_{1}=\varepsilon_{2}=0.1$. Following these assumptions, Fig. 7(b) illustrates how obesity eventually reaches zero in less time than in case (1). This reflects the significance of workouts as well as the role of quitters in the society.

Case (3): We adjust the fast food peer pressure rates to a higher value, that is, $\beta=\gamma=0.2$, but with low quitters' peer pressure rates of $\varepsilon_{1}=\varepsilon_{2}=0.01$. Accordingly, the numerical results in Fig. 7(c) exhibit the eventual disappearance of obesity with high workout rates. However, for low workout rates, obesity remains in the society. This indicates that the low workout rates with high fast food peer pressure rates do not help in minimizing obesity.

Case (4): We set all peer pressure rates to be high, that is, $\beta=\gamma=0.2$ and $\varepsilon_{1}=\varepsilon_{2}=$ 0.1. According to these settings, Fig. 7 (d) displays the time variations of obesity. From the simulations, we find that obesity could be eliminated over time when quitters' peer pressure rates are high. The same applied to workout rates; however, it took longer for obesity to completely disappear with low workout rates than it did with high workout rates.

In summary, the analysis shows that exercise is a significant factor in battling obesity when fast food peer pressure rates are low. However, when these rates are high, then exer- 
cise does not play a major role in diminishing obesity unless workout rates are also high. Moreover, higher values of quitters' peer pressure rates support workout rates in decreasing obesity regardless of the values of fast food peer pressure rates. Furthermore, we find that increasing workout rates reduces the time it took for obesity to be eliminated from society. In conclusion, obesity may be limited by three control measures: first, reducing peer pressure from overweight and obese individuals to consume fast food meals; second, increasing the efforts of quitters in persuading others to avoid fast food meals; and third, maximizing society's awareness of the correlation between exercise and weight loss.

\section{Conclusion}

In this research, two mathematical models were built to examine the distribution of individuals between weight classes based on fast food consumption. The models were represented through a system of nonlinear ordinary differential equations. To understand the resulting behavior, a qualitative approach was utilized by finding the equilibrium points and analyzing their stability. Furthermore, numerical simulations were displayed to support the qualitative results and to analyze critical parameters that suggest how some strategies control obesity.

The first model examined the influence of peer pressure from family members and friends on the average intake of fast food meals. We divided the population into four classes: normal-weight individuals, overweight individuals who ate fast food meals, obese individuals who ate fast food meals, and quitters who no longer eat fast food meals. The results of the qualitative study showed that the system had five equilibrium points. In particular, the free equilibrium point $\left(E_{1,0}\right)$ existed with no conditions and was locally stable when $R_{1,0}<1$. However, if $R_{1,0}>1$, then the overweight equilibrium $\left(E_{1,1}\right)$ existed but was locally stable when $R_{1,1}<1$ and $R_{1,2}<1$. Meanwhile, if $R_{1,1}>1$, then the free quitters equilibrium $\left(E_{1,2}\right)$ existed and was locally stable when $R_{1,1}<R_{1,3}$. Moreover, if $R_{1,2}>1$, then the free obese equilibrium $\left(E_{1,3}\right)$ existed and was locally stable when $R_{1,2}>R_{1,3}$. As for the positive equilibrium $\left(E_{1,4}\right)$, it existed when $0<R_{1,2}<R_{1,3}<R_{1,1}<R_{1,0}$; however, it was globally stable with no conditions. Furthermore, we solved model I numerically and the simulations of the model agreed with the previous qualitative results. Finally, the analysis of the model' parameters suggested that reducing the peer pressure from overweight individuals inflicted on normal-weight individuals had the most significant impact on controlling obesity, along with maximizing the role of quitters in convincing obese individuals to stop consuming fast food meals.

The second model examined the effect of physical activity on reducing weight gain brought on by peer pressure to consume fast food meals. This model was built by adding the exercise factor to model I. The qualitative study of model II produced the same equilibrium points as of model I, but with different existence and local stability conditions. The free equilibrium point $\left(E_{2,0}\right)$ existed with no conditions and was locally stable if $R_{2,0}<1$. However, when $R_{2,0}>1$, then the overweight equilibrium $\left(E_{2,1}\right)$ existed and was locally stable if $R_{2,1}<1$ and $R_{2,2}<1$. On the other hand, when $R_{2,1}>1$, then the free quitters equilibrium $\left(E_{2,2}\right)$ existed and was locally stable if $R_{2,4}<1$. Moreover, when $R_{2,2}>1$, then the free obese equilibrium $\left(E_{2,3}\right)$ existed and was locally stable if $R_{2,5}<1$. Finally, the positive equilibrium $\left(E_{2,4}\right)$ existed when $R_{2,3}>1$ and $\frac{\alpha_{2}+\mu}{\gamma}<y_{2,4}<\frac{\alpha_{3}+\mu}{\varepsilon_{1}}$, but it was locally stable if the eigenvalues of the Jacobian matrix satisfied the Routh-Hurwitz conditions. The research established agreement between the qualitative and numerical results. In addition, 
the analysis of the workout parameters revealed the effects of physical activity in reducing weight.

In conclusion, the results of the mathematical models were consistent with what was observed in the statistical survey (see Sect. 2) as well as in the literature. Thus, the models are suitable for future analysis. From the results, we suggest the following control strategies to combat the development of obesity:

- Emphasizing through education the importance of fighting peer pressure that drives individuals to eat fast food meals;

- Maximizing the effort of quitters in convincing obese individuals to refrain from consuming fast food meals;

- Spreading awareness in the society of the significance of physical activity in preventing weight gain.

\section{Acknowledgements}

Not applicable.

Funding

Not applicable.

Availability of data and materials

Not applicable.

Ethics approval and consent to participate

Not applicable.

Competing interests

The authors declare that they have no competing interests.

Consent for publication

Not applicable.

\section{Authors' contributions}

SA and RM contributed to the design and implementation of the research, to the analysis of the results, and to the writing of the manuscript. All authors read and approved the final manuscript.

\section{Publisher's Note}

Springer Nature remains neutral with regard to jurisdictional claims in published maps and institutional affiliations.

Received: 27 May 2020 Accepted: 5 January 2021 Published online: 21 January 2021

\section{References}

1. Al-Tuwairqi, S.M., Al-Johani, N.O., Simbawa, E.A.: Modeling dynamics of cancer virotherapy with immune response Adv. Differ. Equ. 2020, 438 (2020). https://doi.org/10.1186/s13662-020-02893-6

2. Al-Tuwairqi, S.M., Al-Johani, N.O., Simbawa, E.A.: Modeling dynamics of cancer radiovirotherapy. J. Theor. Biol. 506, 110405 (2020). https://doi.org/10.1016/j.jtbi.2020.110405

3. Al-Tuwairqi, S.M., Bahashwan, W.: A dynamic model of viruses with the effect of removable media on a computer network with heterogeneous immunity. Adv. Differ. Equ. 2020, 260 (2020). https://doi.org/10.1186/s13662-020-02710-0

4. Ali, M.M., Amialchuk, A., Heiland, F.W.: Weight-related behavior among adolescents: the role of peer effects. PLoS ONE 6(6), 1-9 (2011)

5. Anderson, B., Rafferty, A.P., Lyon-Callo, S., Fussman, C., Imes, G.: Fast-food consumption and obesity among Michigan adults. Prev. Chronic Dis. 8(4), A71 (2011)

6. Arenas, A.J., González-Parra, G., Jódar, L.: Periodic solutions of nonautonomous differential systems modeling obesity population. Chaos Solitons Fractals 42(2), 1234-1244 (2009)

7. Arnold, M., Leitzmann, M., Freisling, H., Bray, F., Romieu, I., Renehan, A., Soerjomataram, I.: Obesity and cancer: an update of the global impact. Cancer Epidemiol. 41, 8-15 (2016)

8. Colditz, G.A., Nguyen, N., Dart, H.: Physical Activity and Health. In: International Encyclopedia of Public Health. 463-472 (2017)

9. Diekmann, O., Heesterbeek, J.A., Roberts, M.G.: The construction of next-generation matrices for compartmental epidemic models. J. R. Soc. Interface 7(47), 873-885 (2009)

10. Donnelly, J.E., Hill, J.O., Jacobsen, D.J., Potteiger, J., Sullivan, D.K., Johnson, S.L., Washburn, R.A., et al.: Effects of a 16-month randomized controlled exercise trial on body weight and composition in young, overweight men and women. Arch. Intern. Med. 163(11), 1343 (2003) 
11. Duflou, J., Virmani, R., Rabin, I., Burke, A., Farb, A., Smialek, J.: Sudden death as a result of heart disease in morbid obesity. Am. Heart J. 130(2), 306-313 (1995)

12. Edelstein Keshet, L: Mathematical Models in Biology. SIAM, Philadelphia (2005)

13. Elj, N.E., Lac, G., Tabka, Z., Gharbi, N., Fezaa, S.E.: Effect of physical exercise on reducing food intake and weight gain. Proc., Soc. Behav. Sci. 30, 2027-2031 (2011)

14. Evangelista, A.M., Ortiz, A.R., Rios-Soto, K.R., Urdapilleta, A.: U.S.A. the fast food nation: obesity as an epidemic Technical Reports, Arizona State University (2004)

15. Fortin, B., Yazbeck, M.: Peer effects, fast food consumption and adolescent weight gain. J. Health Econ. 42, 125-138 (2015)

16. Gwozdz, W., Sousa-Poza, A., Reisch, L.A., Bammann, K., Eiben, G., Kourides, Y., Pigeot, ., Peer, I.: Effects on obesity in a sample of European children. Econ. Hum. Biol. 18, 139-152 (2015)

17. Hankinson, A.L., Daviglus, M.L., Bouchard, C., Carnethon, M., Lewis, C.E., Schreiner, P.J., Sidney, S., et al.: Maintaining a high physical activity level over 20 years and weight gain. JAMA 304(23), 2603-2610 (2010)

18. Hirsch, M.W., Smale, S., Devaney, R.L.: Differential Equations, Dynamical Systems and an Introduction to Chaos. Elsevier, Amsterdam (2004)

19. Huang, C., Qiao, Y., Huang, L., et al.: Dynamical behaviors of a food-chain model with stage structure and time delays. Adv. Differ. Equ. 2018, 186 (2018). https://doi.org/10.1186/s13662-018-1589-8

20. Jódar, L., Santonja, F.., González-Parra, G.: Modeling dynamics of infant obesity in the region of Valencia, Spain. Comput. Math. Appl. 56(3), 679-689 (2008)

21. Loh, C.A., Li, Q.: Peer effects in adolescent bodyweight: evidence from rural China. Soc. Sci. Med. 86, 35-44 (2013)

22. Ministry of Health, Kingdom of Saudi Arabia (2013)

23. Nguyen, T., Lau, D.C.: The obesity epidemic and its impact on hypertension. Can. J. Cardiol. 28(3), 326-333 (2012)

24. Nie, P., Sousa-Poza, A., He, X.: Peer effects on childhood and adolescent obesity in China. China Econ. Rev. 35, 47-69 (2015)

25. Niemeier, H.M., Raynor, H.A., Lloyd-Richardson, E.E., Rogers, M.L., Wing, R.R.: Fast food consumption and breakfast skipping: predictors of weight gain from adolescence to adulthood in a nationally representative sample. J. Adolesc Health 39(6), 842-849 (2006)

26. Obesity Update, Organization for Economic Co-operation and Development (2014)

27. Pandit, A., Pandey, A.K.: Obesity context of type 2 diabetes and medication perspectives. Apollo Med. 13(2), 91-96 (2016)

28. Perko, L.: Differential Equations and Dynamical Systems. Springer, Berlin (1991)

29. Pieroni, L., Salmasi, L.: Fast-food consumption and body weight. Evidence from the UK. Food Policy 46, 94-105 (2014)

30. Rosenheck, R.: Fast food consumption and increased caloric intake: a systematic review of a trajectory towards weight gain and obesity risk. Obes. Rev. 9(6), 535-547 (2008)

31. Santonja, F., Morales, A., Villanueva, R., Cortés, J.: Analysing the effect of public health campaigns on reducing excess weight: a modelling approach for the Spanish autonomous region of the community of Valencia. Eval. Program Plann. 35(1), 34-39 (2012)

32. Trogdon, J.G., Nonnemaker, J., Pais, J.: Peer effects in adolescent overweight. J. Health Econ. 27(5), 1388-1399 (2008)

33. Wiklund, P.: The role of physical activity and exercise in obesity and weight management: time for critical appraisal. J. Sport Health Sci. 5(2), 151-154 (2016)

\section{Submit your manuscript to a SpringerOpen ${ }^{\circ}$ journal and benefit from:}

- Convenient online submission

- Rigorous peer review

- Open access: articles freely available online

- High visibility within the field

- Retaining the copyright to your article

Submit your next manuscript at $\boldsymbol{~ s p r i n g e r o p e n . c o m ~}$ 